\title{
GEOMETRIC REALIZATIONS FOR FREE QUOTIENTS
}

\author{
WILLIAM JACO*
}

(Received 6 March 1970)

Communicated by G. E. Wall

\section{Introduction}

In [7] Lyndon introduced the concept of inner rank for groups. He defined the inner rank of an arbitrary group $G$ to be the upper bound of the ranks of free homomorphic images of $G$. Both Lyndon and Jaco have shown that the inner rank of the fundamental group of a closed 2-manifold with Euler characteristic $2-p, p \geqq 0$, is $[p / 2]$ where $[p / 2]$ is the greatest integer $\leqq p / 2$. The proof given by Lyndon [8] uses algebraic techniques; whereas, the proof by Jaco [4] is geometrical.

If the free group $F$ is a homomorphic image of the group $G$, we call $F$ a free quotient of $G$. The purpose of this paper is to give a geometrical interpretation to free quotients of finitely presented groups.

In section 3 we show that whenever the group $G$ can be expressed as a free product $G \approx G_{1} * G_{2}$ where both $G_{1}$ and $G_{2}$ are finitely presented groups, then the inner rank of $G$ is the sum of the inner ranks of $G_{1}$ and $G_{2}$.

\section{Geometric realizations for free quotients}

By $n$-manifold we shall mean a compact connected combinatorial $n$-manifold possibly with boundary $\left[3\right.$, p. 26]. We denote the interior of an $n$-manifold $M^{\mathbf{n}}$ by Int $M^{n}$. The boundary of an $n$-manifold defined as $M^{n}$-Int $M^{n}$ is denoted $\delta M^{n}$. If $\delta M^{n}=\varnothing$, then $M^{n}$ is said to be closed. A $k$-submanifold, $N^{k}$, of the $n$-manifold $M^{n}$ is a $k$-manifold embedded as a subcomplex in some subdivision of $M^{n}$. If $N^{k}$ is a $k$-submanifold of $M^{n}$, we say $N^{k}$ is properly embedded in $M^{n}$ if

$$
N^{k} \cap \delta M^{n}=\delta N^{k} \text {. }
$$

By a surface in the $n$-manifold $M^{n}$, we mean an $(n-1)$-submanifold, $N^{n-1}$, of $M^{n}$ where $N^{n-1}$ is properly embedded in $M^{n}$.

* The author was supported in part by NSF contract GP-11533. 
Let $I$ denote the interval $[-1,1]$. A surface $N^{n-1}$ in $M^{n}$ is said to have a product neighborhood $P\left(N^{n-1}\right)$ if there is a $P L$ embedding

$$
h:\left(N^{n-1} \times I, \delta N^{n-1} \times I\right) \rightarrow\left(M^{n}, \delta M^{n}\right)
$$

so that $h\left(N^{n-1} \times I\right)=P\left(N^{n-1}\right)$ is a neighborhood of $N^{n-1}$ and $h(s \times 0)=s$ for each $s \in N^{n-1}$. The embedding $h$ is called a parametrization of $P\left(N^{n-1}\right)$.

The collection $N_{1}^{n-1}, \cdots, N_{k}^{n-1}$ of surfaces in the $n$-manifold $M^{n}$ is said to be a system of surfaces in $M^{n}$ if

a) $N_{i}^{n-1} \cap N_{j}^{n-1}=\varnothing, i \neq j$, and

b) each $N_{i}^{n-1}$ has a product neighborhood

$$
P\left(N_{i}^{n-1}\right) \text { in } M^{n} \text {. }
$$

A system of surfaces $N_{1}^{n-1}, \cdots, N_{k}^{n-1}$ is called independent if

$$
M^{n}-\bigcup_{i=1}^{k} N_{i}^{n-1}
$$

is connected. Note that whenever $N_{1}^{n-1}, \cdots, N_{k}^{n-1}$ is a system of surfaces in $M^{n}$, then the product neighborhoods $P\left(N_{1}^{n-1}\right), \cdots, P\left(N_{k}^{n-1}\right)$ guaranteed by condition b) may be chosen so that

$$
P\left(N_{i}^{n-1}\right) \cap P\left(N_{j}^{n-1}\right)=\varnothing, \quad i \neq j .
$$

A group $G$ has a free quotient of rank $r$ if there is a homomorphism $\phi$ of $G$ onto a free group of rank $r$.

THEOREM 2.1. Let $M^{n}$ be an $n$-manifold and let $G$ denote the fundamental group of $M^{n}$. Then $G$ has a free quotient of rank $r$ if and only if there is an independent system of surfaces $N_{1}^{n-1}, \cdots, N_{r}^{n-1}$ in $M^{n}$.

PRoOF. Suppose $N_{1}^{n-1}, \cdots, N_{r}^{n-1}$ is an independent system of surfaces in $M^{n}$. Choose product neighborhoods $P\left(N_{1}^{n-1}\right), \cdots, P\left(N_{r}^{n-1}\right)$, one for each $N_{i}^{n-1}$, so that

Then

$$
P\left(N_{i}^{n-1}\right) \cap P\left(N_{j}^{n-1}\right)=\varnothing, \quad i \neq j .
$$

$$
M_{1}^{n}=M^{n}-\bigcup_{i=1}^{r} P^{0}\left(N_{i}^{n-1}\right)
$$

$\left(Y \subset X\right.$, then $Y^{0}$ denotes the point set interior of $Y$ in $\left.X\right)$ is a connected $n$-manifold and $\delta M_{1}^{n}$ contains two copies of $N_{i}^{n-1}$ for each $i=1, \cdots, r$.

Let

$$
h_{i}: N_{i}^{n-1} \times I \rightarrow P\left(N_{i}^{n-1}\right)
$$

denote a parametrization of $P\left(N_{i}^{n-1}\right)$ guaranteed by the definition of $P\left(N_{i}^{n-1}\right)$. 
Choose $s_{i} \in N_{i}^{n-1}$ and let $s_{i j}=h_{i}\left(s_{i} \times j\right)$ for $j=-1,1$. Choose $s_{0} \in \operatorname{Int} M_{1}^{n}$. There are $\operatorname{arcs} \alpha_{i j}, i=1, \cdots, r ; j=-1,1$, embedded in $M_{1}^{n}$ as subcomplexes of some subdivision of $M_{1}^{n}$ so that

a) $\alpha_{i j}$ is an arc from $s_{0}$ to $s_{i j}$,

b) $\alpha_{i j} \cap \alpha_{k l}=\left\{s_{0}\right\},(i, j) \neq(k, l)$, and

c) $\alpha_{i j}-\left\{s_{i j}\right\} \subset \operatorname{Int} M_{1}^{n}$.

Let $\Gamma^{\prime}=\bigcup_{i, j} \alpha_{i j}$. Then $\Gamma^{\prime}$ is a wedge at $s_{0}$ of the arcs $\alpha_{i j}$. There is a retraction $f_{1}$ of $P\left(N_{i}^{n+1}\right)$ onto $h_{i}\left(s_{i} \times I\right)$. Let $f_{2}^{\prime}$ denote the retraction of

onto $\Gamma^{\prime}$ defined as

$$
\Gamma^{\prime} \cup \bigcup_{i, j} h_{i}\left(N_{i}^{n-1} \times\{j\}\right), \quad j=-1,1
$$

$$
\begin{gathered}
f_{2}^{\prime}\left|\Gamma^{\prime}=\mathrm{id}\right| \Gamma^{\prime}, \text { and } \\
f_{2}^{\prime}\left|\bigcup_{i, j} h_{i}\left(N_{i}^{n-1} \times\{j\}\right)=f_{1}\right| \bigcup_{i, j} h_{i}\left(N_{i}^{n-1} \times\{j\}\right) .
\end{gathered}
$$

Then by Tietze's Theorem [2], there is an extension $f_{2}$ of $f_{2}^{\prime}$ retracting $M_{1}^{n}$ onto $\Gamma^{\prime}$.

Let $\Gamma$ be the wedge of $r$ simple closed curves defined as

$$
\Gamma=\Gamma^{\prime} \cup \bigcup_{i} h_{i}\left(s_{i} \times I\right) \text {. }
$$

The map $f: M^{n} \rightarrow \Gamma$ defined as

$$
\begin{gathered}
f\left|\bigcup_{i} P\left(N_{i}^{n-1}\right)=f_{1}\right| \bigcup_{i} P\left(N_{i}^{n-1}\right) \text { and } \\
f\left|M_{1}^{n}=f_{2}\right| M_{1}^{n}
\end{gathered}
$$

is a retraction of $M^{n}$ onto $\Gamma$. Hence, $G$ has a free quotient of rank $r$.

Choose a point $s_{0} \in \operatorname{Int} M^{n}$. Suppose $\psi$ is a homomorphism of $\pi_{1}\left(M^{n}, s_{0}\right)$ onto $F$, the free group of rank $r$. We are interested in the case $r \geqq 1$.

Let $T$ denote a wedge at $t_{0}$ of $r$ simple closed curves $T_{1}, \cdots, T_{r}$. Then there is a homomorphism $\phi$ of $\pi_{1}\left(M^{n}, s_{0}\right)$ onto $\pi_{1}\left(T, t_{0}\right)$. Let $f$ denote a simplicial map of some subdivision of $M^{n}$ to some subdivision of $T$ taking $s_{0}$ to $t_{0}$ so that the homomorphism $f_{*}$ of $\pi_{1}\left(M^{n}, s_{0}\right)$ to $\pi_{1}\left(T, t_{0}\right)$ induced by $f$ is equal to $\phi$.

Choose points $t_{i}, 1 \leqq i \leqq r$, so that $t_{i}$ is interior to some 1 -simplex, $\Delta_{i}$, of $T_{i}$ in the subdivision of $T$ for which $f$ is simplicial. Let $\delta_{i}: t_{i} \times I$ be a linear embedding of $t_{i} \times I$ into $\Delta_{i}^{0}$ so that $\delta_{i}\left(t_{i} \times 0\right)=t_{i}$. Then $U_{i} f^{-1}\left(t_{i}\right)$ is a system of surfaces in $M^{n}$. Furthermore, for any surface $N_{i k}^{n-1} \in f^{-1}\left(t_{i}\right)$ a product neighborhood $P\left(N_{i k}^{n-1}\right)$ of $N_{i k}^{n-1}$ may be chosen so that $P\left(N_{i k}^{n-1}\right)$ has a parametrization

$$
h_{i k}: N_{i k}^{n-1} \times I \rightarrow P\left(N_{i k}^{n-1}\right)
$$

where for each $s \in N_{i k}^{n-1}, h_{i k}(s \times I)$ is carried by the map $f$ both homeomorphically and linearly onto $\delta_{i}\left(t_{i} \times I\right)$ and 


$$
P\left(N_{i k}^{n-1}\right) \cap P\left(N_{l m}^{n-1}\right)=\phi, \quad \text { if }(i, k) \neq(l, m) .
$$

Let $S_{i}$ denote the system of surfaces $f^{-1}\left(t_{i}\right)$.

Let $U_{i}=\delta_{i}\left(t_{i} \times I\right)$. We call $U_{i}^{+}=\delta_{i}\left(t_{i} \times[0,1]\right)\left(U_{i}^{-}=\delta_{i}\left(t_{i} \times[-1,0]\right)\right)$ the positive side (negative side) of $U_{i}$. We call

$$
P_{+}\left(N_{i k}^{n-1}\right)=h_{i k}\left(N_{i k}^{n-1} \times[0,1]\right)\left(P_{-}\left(N_{i k}^{n-1}\right)=h_{i k}\left(N_{i k}^{n-1} \times[-1,0]\right)\right)
$$

the positive side (negative side) of $P\left(N_{i k}^{n-1}\right)$.

Let $\bar{t}_{i}$ denote an embedding of $S^{1}$ onto $T_{i}$ so that if we think of the class of $\bar{t}_{i}$ in $\pi_{1}\left(T, t_{0}\right), \bar{t}_{i}$ is oriented so as to 'cross' $t_{i}$ from $U_{i}^{-}$to $U_{i}^{+}$. We also write $\bar{t}_{i}$ for the class of $\bar{t}_{i}$ in $\pi_{1}\left(T, t_{0}\right)$.

Since the homomorphism

$$
f_{*}: \pi_{1}\left(M^{n}, s_{0}\right) \rightarrow \pi_{1}\left(T, t_{0}\right)
$$

induced by $f$ is onto, there is a loop $l_{i}, 1 \leqq i \leqq r$, in $M^{n}$ based at $s_{0}\left(l_{i}\right.$ may be chosen as a simple closed curve if $n \geqq 3$ ) so that the element $\left[l_{i}\right]$ of $\pi_{1}\left(M^{n}, s_{0}\right)$ determined by $l_{i}$ is carried onto $\bar{t}_{i}$ by $f_{*}$. We may assume that $l_{i}$ is chosen in general position with respect to $\bigcup_{i} S_{i}$.

There is a procedure $\left[4\right.$, p. 368] for reading the word $w\left(l_{i}\right)$ in the symbols $\bar{t}_{1}, \bar{t}_{1}^{-1}, \cdots, \bar{t}_{r}, \bar{t}_{r}^{-1}$ of the free group $\pi_{1}\left(T, t_{0}\right)$ which corresponds to $f_{*}\left[l_{i}\right]$ by observing the way $l_{i}$ meets $\bigcup_{i} S_{i}$. Since $\pi_{1}\left(T, t_{0}\right)$ is a free group and $w\left(l_{i}\right)$ is equal to $\tilde{t}_{i}$ in $\pi_{1}\left(T, t_{0}\right)$, it must be true that either $l_{i}$ meets only one component of $S_{i}$ or that there is a cancellation of the form $\bar{t}_{j} \bar{t}_{j}^{-1}$ (or $\bar{t}_{j}^{-1} \bar{t}_{j}$ ) in $w\left(l_{i}\right)$.

At this point it is convenient to consider the two cases $n<3$ and $n \geqq 3$.

CASE 1. $n<3$. If $n=1$, then a separate, straightforward argument applies. If $n=2$, then the desired conclusion is just Lemma 3.2. of [4].

CASE 2 . $n \geqq 3$. We now have that $l_{i}, 1 \leqq i \leqq r$, is a simple closed curve in $M^{n}$ based at $s_{0}$ and $l_{i} \cap l_{j}=\left\{s_{0}\right\}, i \neq j$. A cancellation of the form $\bar{t}_{j} \bar{t}_{j}^{-1}$ (or $\left.\bar{t}_{j}^{-1} \bar{t}_{j}\right)$ in $w\left(l_{i}\right)$ has as its geometric counterpart in $M^{n}$ a subarc $\alpha_{i}$ of $l_{i}$ which meets $\bigcup_{i} S_{i}$ only in its end points which are both in $S_{j}$ (possibly not the same component of $S_{j}$ ). We shall use this geometric interpretation of the reduction of $w\left(l_{i}\right)$ to $\bar{t}_{i}$ in $\pi_{1}\left(T, t_{0}\right)$ to obtain a system of surfaces $N_{1}^{n-1}, \cdots, N_{r}^{n-1}$ in $M^{n}$ so that $l_{i} \cap N_{i}^{n-1}$ is precisely one point at which $l_{i}$ pierces $N_{i}^{n-1}$; i.e. $l_{i}$ meets both $P_{+}\left(N_{i}^{n-1}\right)$ and $P_{-}\left(N_{i}^{n-1}\right)$. Furthermore, $l_{i} \cap N_{j}^{n-1}$ will be void for $i \neq j$.

Consider the subarc $\alpha_{i}$ of $l_{i}$ with its endpoints in $S_{j}$ and otherwise $\alpha_{i}$ misses $\bigcup_{i} S_{i}$. There is a combinatorial $n$-cell $Q^{n} \subset M^{n}$ and parametrization of $Q^{n}$ as a product of the combinatorial $(n-1)$-cell $Q^{n-1}$ and the interval $I$ so that

a) for some point $0 \in \operatorname{Int} Q^{n-1}$,

$$
\alpha_{i}=0 \times I,
$$

b) $Q^{n} \cap l_{i}=\alpha_{i}, Q^{n} \cap l_{j}=\varnothing, i \neq j$, 
c) $Q^{n} \cap S_{i}=\varnothing, i \neq j$,

d) $Q^{n}$ meets only the components of $S_{j}$ which $\alpha_{i}$ meets, and

e) $Q^{n} \cap S_{j}=Q^{n-1} \times-1 \cup Q^{n-1} \times 1$.

Let $S_{j}^{\prime}$ be the system of surfaces in $M^{n}$ obtained from $S_{j}$ and $\mathrm{Bd} Q$ by replacing the $(n-1)$-cells $Q^{n-1} \times-1$ and $Q^{n-1} \times 1$ by the closed annulus $\delta Q^{n-1} \times I$.

We now have a collection $S_{1}^{\prime}, \cdots, S_{j}^{\prime}, \cdots, S_{r}^{\prime}$ where each $S_{i}^{\prime}$ is a system of surfaces in $M^{n}, S_{i}^{\prime}=S_{i}, i \neq j$ and $S_{j}^{\prime}$ is described above. The word problem for the simple closed curve $l_{i}$ has been reduced with respect to this collection since the cancellation $\bar{t}_{j} \bar{t}_{j}^{-1}$ (or $\bar{t}_{j}^{-1} \bar{t}_{j}$ ) has been eliminated as viewed geometrically. In other words we have reduced the number of components of $l_{i} \cap \bigcup_{i} S_{i}$ by looking at the number of components of $l_{i} \cap \bigcup_{i} S_{i}^{\prime}$. In a finite number of steps, we obtain the system of surfaces $N_{1}^{n-1}, \cdots, N_{r}^{n-1}$ in $M^{n}$ promised above.

Since the wedge of simple closed curves $\bigcup_{i} l_{i}$ has the property $l_{i} \cap N_{i}^{n-1}$ is precisely one piercing point and $l_{i} \cap N_{j}^{n-1}=\varnothing, i \neq j$, the system of surfaces $N_{1}^{n-1}, \cdots, N_{r}^{n-1}$ is independent in $M^{n}$. This concludes the proof of Proposition 2.1.

\section{Additivity of inner rank}

Suppose $G_{1}$ and $G_{2}$ are groups. We designate the free product of $G_{1}$ and $G_{2}$ by $G_{1} * G_{2}[6]$.

To this author's knowledge a result like the following lemma first appeared in the literature in [9]. We include a brief outline of a proof for completeness of our argument.

Lemma 3.1. Suppose $G$ is a finitely presented group. Then there is a closed, connected, combinatorial 4-manifiold $M_{G}^{4}$ so that $\pi_{1}\left(M_{G}^{4}\right) \approx G$.

Proof. There is a connected, finite, simplicial 2-complex $K_{G}$ with $\pi_{1}\left(K_{G}\right) \approx G$ [1, Theorem 6.4.6]. Let $h$ denote a simplicial embedding of $K_{G}$ into a standard rectilinear subdivision of the 5-sphere, $S^{5}$.

If $N\left(K_{G}\right)$ denotes a regular neighborhood of $h\left(K_{G}\right)$ in $S^{5}[3$, p. 59], then

$$
M_{G}^{4}=\delta N\left(K_{G}\right)
$$

is the desired closed, connected, combinatorial 4-manifold.

Suppose $G$ is a group. Let

$$
I N(G)=\max _{F} r(F)
$$

where $F$ is a free quotient of $G$ and $r(F)$ denotes the rank of $F$. We call $I N(G)$ the inner rank of $G$.

THEOREM 3.2. Suppose $G_{1}$ and $G_{2}$ are both finitely presented groups.

$$
I N\left(G_{1} * G_{2}\right)=I N\left(G_{1}\right)+I N\left(G_{2}\right) \text {. }
$$


Proof. Let $M_{1}^{4}, M_{2}^{4}$ denote closed, 4-manifolds where $\pi_{1}\left(M_{1}^{4}\right) \approx G_{1}$ and $\pi_{1}\left(M_{2}^{4}\right) \approx G_{2}$. Let $r_{1}=I N\left(G_{1}\right), r_{2}=I N\left(G_{2}\right)$.

There is a homomorphism $\phi$ of $G_{1} * G_{2}$ onto the free group $F$ of rank $r_{1}+r_{2}$. Hence

$$
I N\left(G_{1} * G_{2}\right) \geqq I N\left(G_{1}\right)+I N\left(G_{2}\right) .
$$

Let $M^{4}$ denote the closed, connected combinatorial 4-manifold $M_{1}^{4} \not M_{2}^{4}$ obtained from $M_{1}^{4}$ and $M_{2}^{4}$ via connected sum. Then by Van Kampen's Theorem we have

$$
\pi_{1}\left(M^{4}\right) \approx G_{1} * G_{2}
$$

If $F$ is a free group of rank $s$ and $\phi$ is a homomorphism of $G_{1} * G_{2}$ onto $F$, then by Proposition 2.1 there is an independent system of surfaces $N_{1}^{3}, \cdots, N_{s}^{3}$ in $M^{4}$.

There is a 3-sphere $S^{3} \subset M^{4}$ so that

a) $M^{4}-S^{3}$ has precisely two components $Q_{1}^{4}, Q_{2}^{4}$ where the closure of $Q_{i}^{4}$ is $P L$ homeomorphic with $M_{i}^{4}$ minus the interior of a 4-cell in $M_{i}^{4}, i=1,2$, and

b) $\bigcup_{i=1}^{s} N_{i}^{3} \cap S^{3}=\bigcup_{j=1}^{p} F_{j}^{2}$ where $F_{1}^{2}, \cdots, F_{p}^{2}$ is a system of surfaces in $S^{3}$ (possible an empty collection).

Let $p$, the number of components of

$$
\bigcup_{i} N_{i}^{3} \cap S^{3}
$$

denote the complexity of the system $N_{1}^{3}, \cdots, N_{s}^{3}$ relative to $S^{3}$. If $p=0$, then it follows that $s \leqq r_{1}+r_{2}$ and thus

$$
I N\left(G_{1} * G_{2}\right) \leqq I N\left(G_{1}\right)+I N\left(G_{2}\right) .
$$

If $p>0$, then we shall show that there is an independent system of surfaces $R_{1}^{3}, \cdots, R_{s}^{3}$ in $M^{4}$ where the complexity of the system $R_{1}^{3}, \cdots, R_{s}^{3}$ relative to $S^{3}$ is strictly less than $p$.

There is a product neighborhood $P\left(S^{3}\right)$ of $S^{3}$ in $M^{4}$ and a parametrization

so that

$$
h: S^{3} \times I \rightarrow P\left(S^{3}\right)
$$

$$
\bigcup_{i=1}^{s} N_{i}^{3} \cap P\left(S^{3}\right)=\bigcup_{j=1}^{p} h\left(F_{j}^{2} \times I\right) .
$$

Actually, we may have to move the system $N_{1}^{3}, \cdots, N_{s}^{3}$ by an ambient homeomorphism to obtain an independent system which satisfies this condition. Since such a homeomorphism may be chosen so as to leave $S^{3}$ invariant, we continue to use the same notation for this new system. 
Each $F_{j}^{2}$ separates $S^{3}$. Choose the indexing of $F_{1}^{2}, \cdots, F_{p}^{2}$ so that $F_{p}^{2}$ is an innermost surface in $S^{3}$; i.e. one of the two domains complementary to $F_{p}$ in $S^{3}$ meets no $F_{j}^{2}$. Let $D$ denote the closure of this domain in $S^{3}$. Suppose $F_{p}^{2} \subset N_{k}^{3} \cap S^{3}$. There are two cases to consider:

CASE 1. $F_{p}^{2}$ does not separate $N_{k}^{3}$. Let

$$
R_{k}^{3}=\left(N_{k}^{3}-h\left(F_{p}^{2} \times I\right)\right) \cup(h(D \times\{1\}) \cup h(D \times\{-1\})) .
$$

Then $R_{1}^{3}, \cdots, R_{k}^{3}, \cdots, R_{s}^{3}$ defined as $R_{i}^{3}=N_{i}^{3}, i \neq k$, is an independent system of surfaces in $M^{4}$ and the complexity of the system $R_{1}^{3}, \cdots, R_{s}^{3}$ is $p-1$.

CASE 2. $F_{p}^{2}$ separates $N_{k}^{3}$. Let $N_{k_{1}}^{3}$ and $N_{k_{2}}^{3}$ denote the closure in $N_{k}^{3}$ of the two components complementary to $F_{p}^{2}$ in $N_{k}^{3}$. Since $N_{1}^{3}, \cdots, N_{k}^{3}$ is an independent system of surfaces in $M^{4}$, there is a wedge $T$ of a simple curves $T_{1}, \cdots, T_{s}$ in $M^{4}$ so that $T_{i} \cap N_{i}^{3}$ is a single piercing point and $T_{i} \cap N_{j}^{3}=\varnothing, i \neq j$. Furthermore, $T \cap D=\varnothing$. Choose notation so that

$$
T \cap N_{k}^{3}=T \cap N_{k_{1}}^{3} \text {. }
$$

Suppose

$$
N_{k_{1}}^{3} \cap P\left(S^{3}\right) \supset h\left(F_{p}^{2} \times\{1\}\right)
$$

Let

$$
R_{k}^{3}=\left(N_{k_{1}}^{3}-h\left(F_{p}^{2} \times I\right)\right) \cup h\left(F_{p}^{2} \times\{1\}\right) .
$$

Then $R_{1}^{3}, \cdots, R_{k}^{3}, \cdots, R_{s}^{3}$ defined as $R_{i}^{3}=N_{i}^{3}, i \neq k$ is an independent system of surfaces in $M^{4}$. For $p^{\prime}$ the complexity of the system $R_{1}^{3}, \cdots, R_{s}^{3}$ we have $p^{\prime} \leqq p-1$.

We conclude that for $N_{1}^{3}, \cdots, N_{s}^{3}$ any independent system of surfaces in $M^{4}$, that there is an independent system of surfaces $R_{1}^{3}, \cdots, R_{s}^{3}$ in $M^{4}$ where the complexity of the system $R_{1}^{3}, \cdots, R_{s}^{3}$ relative to $S$ is zero. By the remark above, this completes the proof of Theorem 3.2. Lyndon has shown the author an algebraic proof of Theorem 3.2.

Several applications of Theorem 3.2 are given in [5].

\section{References}

[1] P. J. Hilton and S. Wylie, Homology Theory (Camb. Univ. Press, Cambridge, 1962).

[2] J. G. Hocking and G. S. Young, Topology, (Addison-Wesley, Reading, Mass., 1961).

[3] J. E. P. Hudson, Piecewise Linear Topology (W. A. Benjamin, New York, 1969).

[4] W. Jaco, 'Heegaard splitting and splitting homomorphisms', Trans. A. M.S., Vol. 146 (1969), 365-375.

[5] W. Jaco, 'Non-retractible cubes-with-holes', Michigan Math. J., Vol. 18 (1971), 193-201.

[6] A. G. Kurosh, The Theory of Groups, Vol. 1, II (Chelsea, New York, N.Y., 1960). 
[7] R. C. Lyndon, 'The equation $a^{2} b^{2}=c^{2}$ in free groups', Mich. Math. J. 6 (1959), 89-95.

[8] R. C. Lyndon, 'Dependence in groups,' Colloq, Mathe. (Warsaw) XIV (1966), 275-283.

[9] A. Markov, 'The insolubility of the problem of homeomorphy', Dokl. Akad. Nauk SSSR 121 (1958), 218-220.

Department of Mathematics

Rice University

Houston, Texas

U.S.A. 